\title{
ALFALFA in the Leo Region: Looking for Missing Satellites in HI
}

\author{
Sabrina Stierwalt ${ }^{1}$ \\ ${ }^{1}$ Center for Radiophysics and Space Research, Cornell University, Ithaca, NY 14853, \\ email: sabrina@astro.cornell.edu
}

\begin{abstract}
The location of two nearby galaxy groups within $\sim 20 \mathrm{Mpc}$ in the Leo region allows for a detailed study of low-mass galaxies. A catalog of HI line detections in Leo $(9 h 36 m<\alpha<$ $11 \mathrm{~h} 36 \mathrm{~m},+8^{\circ}<\delta<+16^{\circ}$ ) has been made from the blind HI survey ALFALFA. More sensitive single-pixel Arecibo observations targeted Leo dwarf candidates noted optically by Karachentsev et al. 2004 (K04) to determine group members and allow for a comparison of HI and opticallyselected samples. This presentation highlights the differences between the two samples and the significant contribution blind HI surveys can make to the missing satellites problem.
\end{abstract}

Keywords. galaxies: distances and redshifts, galaxies: dwarf, galaxies: evolution, galaxies: formation, galaxies: mass function, galaxies: spiral, (cosmology:) cosmological parameters, cosmology: observations, (cosmology:) large-scale structure of universe, radio lines: galaxies

\section{The ALFALFA Survey of the Leo Region}

As described by Giovanelli, Haynes and others in these proceedings, the blind HI survey Arecibo Legacy Fast ALFA (ALFALFA) utilizes the Arecibo Observatory's new 7 -beam feed array to map $7000 \mathrm{deg}^{2}$ of sky out to $\mathrm{cz} \sim 18,000 \mathrm{kms}^{-1}$. One of the first areas to be covered in full by ALFALFA was the Leo region, and in these $240 \mathrm{deg}^{2}$ of sky ALFALFA has produced roughly 1300 good quality HI detections, $75 \%$ of which are new HI detections. Roughly 40 are found to have HI masses $<10^{7.5} M_{\odot}$. Complex structure in velocity space at $\mathrm{cz}<3000 \mathrm{kms}^{-1}$ forms 2 distinct entities, the Leo I and Leo II groups. The nearer Leo I group (also the M96 group) is placed at $10.4 \mathrm{Mpc}$ using Cepheid variables and surface brightness fluctuations. Leo I offers a uniquely intermediate density environment with both a low velocity dispersion $(\sim 130 \mathrm{~km} / \mathrm{s})$ and a local density enhancement high enough to support E/S0 galaxies. Within Leo I exists further substructure including a ring of intergalactic gas $\sim 200 \mathrm{kpc}$ in diameter surrounding the M96 group (Schneider et al. 1983), and a long tidal stream of stars and gas produced by a galaxy interaction in the Leo Triplet. Leo I also differs from most nearby groups in its comparatively larger distance of 3-4 Mpc from the supergalactic plane (K04).

\section{HI and Optical Comparisons}

A smaller region from $10 h 30 m$ to $11 \mathrm{~h} 5 \mathrm{~m}$ in RA was surveyed by K04, and potential Leo members were selected via a visual inspection of POSS-II/ESO/SERC plates. To verify group members and create an optically-selected catalog of Leo dwarfs, single-pixel Arecibo observations were taken of the identified candidates. Twenty of a possible 35 dwarfs were detected in the high-sensitivity HI observations, including 5 background sources, with integration times from 2 to 16 min on-source (compared to ALFALFA's $\sim 40 \mathrm{sec} /$ beam). Two detections lie in the direction of the Leo Ring and thus require further analysis to determine the relationship of the stellar dwarf to the detected HI. 


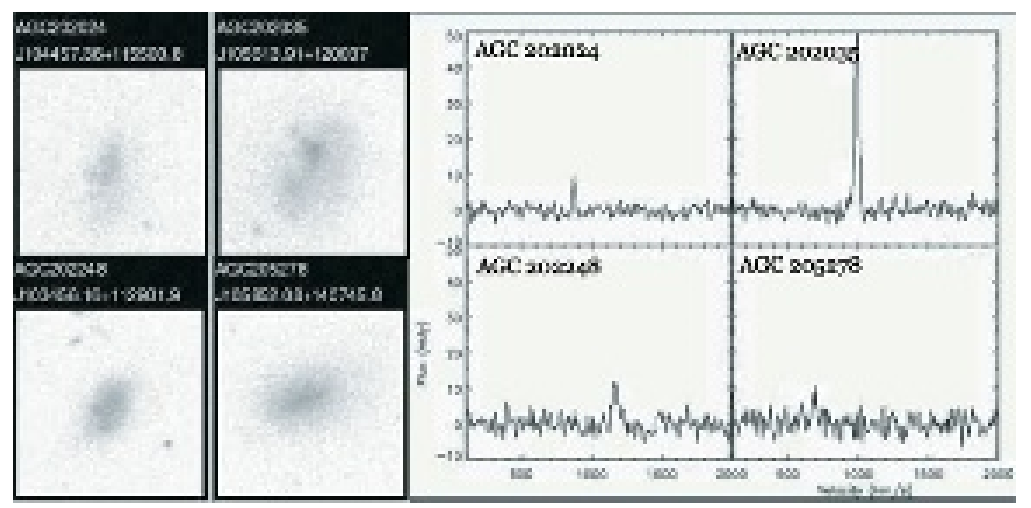

Figure 1. SDSS images (50" scale) of 4 Leo dwarfs with their ALFALFA detections. AGC202024 \& AGC202035 were noted in K04's optically-selected catalog while AGC202248 \& AGC205278 were not. All have HI masses $<10^{8} \mathrm{M}_{\odot}$.

Only three of the optically-selected objects detected in the targeted HI observations were not detected in ALFALFA because their fluxes lie below ALFALFA's detection limit. An additional $\sim 20$ probable Leo dwarfs were newly identified in the HI catalog. Four examples of these low-mass objects are shown in Figure 1. When placed at the group distance of $10.4 \mathrm{Mpc}$, AGC202248 and AGC202035 have HI masses less than $10^{8} M_{\odot}$ $\left(\sim 10^{7.21} M_{\odot}\right.$ and $10^{7.63} M_{\odot}$ respectively), while AGC202024 and AGC205278 are even dwarfier with HI masses less than $10^{7} M_{\odot}\left(\sim 10^{6.71} M_{\odot}\right.$ and $10^{6.94} M_{\odot}$ respectively $)$.

\section{Conclusions and Future Work}

The ALFALFA survey has been very effective in finding gas-rich dwarfs that have previously been overlooked by optical surveys. With the completion of the ALFALFA Leo catalog, the origins of these dwarfs can now be investigated. The gas-rich dwarfs most commonly found in ALFALFA are often optically blue \& clumpy, and many of these dwarfs are very low surface brightness making star formation activity difficult to determine optically. In such cases sites of star formation can often still be identified by the faint blue light the youngest stars emit in the ultraviolet. GALEX observations of the lowest-mass Leo dwarfs are currently underway, and the next stage of this project will be to compare sites of recent star formation with HI column densities.

\section{Acknowledgements}

This work has been supported by NSF grants AST-0307661, AST-0435697 and AST0607007 and by the Brinson Foundation. Arecibo Observatory is part of the NAIC which is operated by Cornell University under a cooperative agreement with the NSF. Funding for SDSS has been provided by the Alfred P. Sloan Foundation, the Participating Institutions, NSF, US DoE, NASA, the Japanese Monbukagakusho, the Max Planck Society, and the Higher Education Funding Council for England. The SDSS Web site is http://www.sdss.org/.

\section{References}

Giovanelli, R et al.. 2007, Astro.J. 133, 2569

Giovanelli et al. 2005a, Astro.J. 130, 2598

Karachentsev, Karachentseva, Huchtmeier, \& Makarov 1994, Astro.J. 127, 2031

Schneider, Helou, Salpeter, \& Terzian 1983, Ap.J.(Lett) 273, L1 